\title{
PARÂMETROS POPULACIONAIS DE Sagitta friderici Ritter-Záhony (CHAETOGNATHA) NA ZONA DE ARREBENTAÇÃO DA PRAIA DO CASSINO, SUL DO BRASIL
}

\author{
CHARRID RESGALLA JR. \\ Universidade do Vale do Itajaí (UNIVALI), Centro de Ensino em Ciências Tecnológicas da Terra e do Mar (CTTMar), Cx. Postal 360 \\ CEP-88.302-202 Itajaí, SC, Brasil. cresgalla@univali.br
}

\begin{abstract}
Foram analisadas 36 amostras de zooplâncton obtidas entre 10 de outubro de 1990 a 24 de abril de 1992 em um ponto

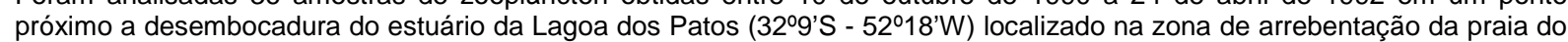
Cassino. Dados de densidade, biomassa, variação de comprimento e a distrubuição de freqüência dos estágios de desenvolvimento do Chaetognatha Sagitta friderici foram obtidos para conhecer as características populacionais deste importante predador na cadeia pelágica zooplanctônica. A população de $S$. friderici apresentou 3 picos de densidade ao longo do ano e uma oscilação de comprimento dos diferentes estágios de desenvolvimento que responderam as variações da temperatura da água. Foram identificados 3 coortes ao longo do ano com base na ocorrência do estágio IV de desenvolvimento (adultos em processo de reprodução) seguidos pelo estágio 0 (juvenis) nas amostras. Os tempos de desenvolvimento foram de 73 dias para a coorte de Primavera/Verão, 95 dias para Verão/Outono e 136 dias para Outono/Primavera, assim como uma relação inversa com a temperatura média registrada para cada período. A população de $S$. friderici da zona de arrebentação da praia do Cassino, sul do Brasil, apresenta características similares as observadas na população do estuário de Cananéia, litoral
\end{abstract} de São Paulo.

PALAVRAS CHAVE: Zooplâncton, Praia intermediária/dissipativa, Tempo de desenvolvimento, Coorte, Biomassa. \section{ABSTRACT
Population parameters of Sagitta friderici Ritter-Záhony (Chaetognatha) in the surf zone of Cassino Beach,
South of Brazil}

An analysis was carried out of 36 samples of zooplankton obtained between 10th October 1990 and 24th April 1992 at a station close to the mouth of the Lagoa dos Patos estuary $\left(32^{\circ} 9^{\prime} \mathrm{S}-52^{\circ} 18^{\prime} \mathrm{W}\right)$ located in the surf zone of Cassino beach. Data on density, biomass, size variation and frequency distribution of the development stages the Chaetognatha Sagitta friderici were obtained, in order to determine the population characteristics of this major predator in the zooplankton pelagic chain. The population of $S$. friderici presented three density peaks during the year, and a size variation during the different development stages which reflected the variations in water temperature. Three cohorts were identified over the year, based on the occurrence of developmental stage 4 (mature adults), followed by stage 0 (juveniles) in the samples. Development took 73 days for the Spring/Summer cohort, 95 days for the Summer/Fall and 136 days for the Fall/Spring. The population of $S$. friderici of the surf zone of Cassino beach, in the South of Brazil, presented similar characteristics with Cananéia estuary population, in the coastal area of São Paulo.

KEYWORDS: Zooplankton, Intermediary/dissipative beach, Development time, Cohort, Biomass.

\section{INTRODUÇÃO}

Em amostras do zooplâncton marinho, o filo Chaetognatha normalmente apresenta-se com uma alta frequência de ocorrência e abundância, perfazendo, normalmente, de 10 a $30 \%$ da biomassa de copépodes (Bone et al. 1991). Carnívoros oportunistas, os quetognatos desempenham um importante papel na comunidade pelágica, agindo, entre outros fatores, como consumidores de copépodes e como competidores e predadores diretos sobre o ictioplâncton. São também excelentes indicadores hidrográficos (Boltovskoy 1981) e um dos grupos mais bem estudados da costa brasileira (Lopes 2007).

Para os ambientes no litoral dos estados do sul do Brasil, como a baía de Paranaguá (Montú \& Cordeiro 1988), desembocadura do estuário do rio Itajaí-açu (Resgalla Jr. et al. 2008), estuário da Lagoa dos Patos (Montú 1980), praias arenosas expostas do município de Rio Grande (Bersano 1994) e plataforma continental (Resgalla Jr. \& Montú 1995), Sagitta friderici ( = Sagitta tenuis) é a espécie de quetognata dominante, ocorrendo durante todo o ano. No sul do Brasil, durante o inverno é a única espécie encontrada nestes ambientes, enquanto que no verão, devido a maior influência da Corrente do Brasil, Sagitta enflata e Sagitta hispida constituem as outras espécies da associação de águas costeiras quentes (Resgalla Jr. 2008). Na costa do Rio Grande do Sul, S. friderici delimita a influência da água costeira (salinidade $<30$ ) tanto de características termófilas como criófilas. $\mathrm{Na}$ costa da Argentina esta espécie também é dominante, caracterizando a água de plataforma desta região (Cavalieri 1963, Ramirez \& Viñas 1982).

Devido a sua importância no ecossistema planctônico, e com o objetivo de conhecer alguns parâmetros populacionais da espécie, foram estudados as variações morfológicas, estágios de maturação e a distribuição da freqüência das classes de comprimento ao longo de 19 meses de coletas 
realizadas na zona de arrebentação da praia do Cassino, extremo sul do Brasil, para comparações com populações encontradas em outras regiões.

\section{ÁREA DE ESTUDO}

A praia do Cassino é um extenso segimento praial arenoso, com características de praia intermediária a dissipativa (Borzone 1988), localizada no extremo sul do Brasil (32 ${ }^{\circ} \mathrm{S}$ a $\left.34^{\circ} \mathrm{S}\right)$ (Fig. 1). A zona de arrebentação, porção do infralitoral limitada entre a primeira linha de quebra das ondas e o limite inferior do médiolitoral, é caracterizada por uma área de alta turbulência. A sua largura é variável e dependente da energia de onda, podendo ser inexistente em condições de calmaria e com até 500 $\mathrm{m}$ de distância da costa em condições de ondas superiores a $3 \mathrm{~m}$ de altura, mas normalmente apresentam uma largura de 50 a 150 m (Borzone 1988).

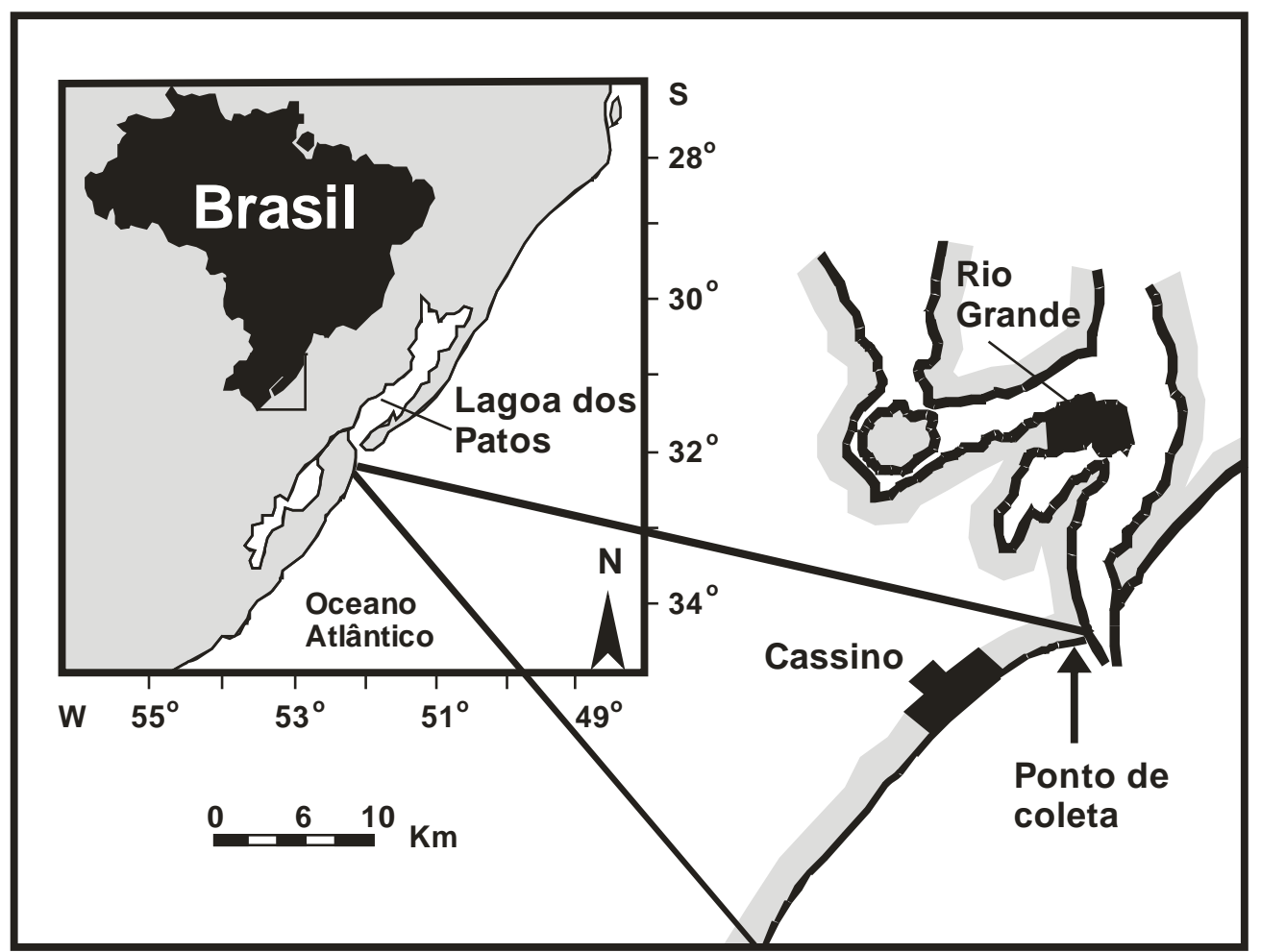

Figura 1 - Localização da praia do Cassino no sul do Brasil e ponto de coleta das amostras de zooplâncton.

O sistema de circulação litorânea é determinado pelas condições meteorológicas, principalmente pelo regime de ventos, já que a amplitude de maré é pouco significativa na região (Calliari 1997). A corrente de deriva litorânea, paralela a linha de costa, originária da incidência obliqua das ondas é a principal dinâmica das águas. Apesar do domínio dos ventos de NE, as correntes de deriva de sul para o norte são principalmente observados e ocasionados por ondulações de SE (Bersano 1994).

As condições oceanográficas da plataforma adjacente é influenciada pela Água Tropical de plataforma nos meses quentes (Castello \& Möller Jr. 1977) e pela frente do Prata, constituída pela mistura entre a Água Subantártica da corrente das Malvinas com a descarga do rio do Prata nos meses frios (Piola et al. 2000). Por outro lado, o aporte do estuário da Lagoa dos Patos apresenta forte influência sobre a área de estudo (Borzone 1988).

\section{MATERIAL E MÉTODOS}

As amostras das análises quantitativas e da distribuição de comprimento de Sagitta friderici foram obtidas através de coletas quinzenais entre 10 de outubro de 1990 e 24 de abril de 1992. O ponto de coleta selecionado foi próximo ao molhe oeste da

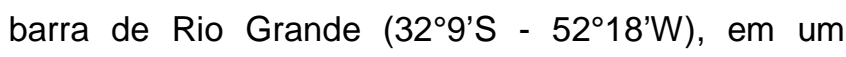
segmento de praia arenosa (Fig. 1) e dentro da zona de arrebentação. Este ponto foi escolhido por 
apresentar características hidrodinâmicas diferenciadas, com 0 acúmulo de material em suspensão proporcionado por derivas litorâneas na maior parte do ano (Bersano 1994).

As amostras foram obtidas por arrastos horizontais de 2 minutos de duração mediante o uso de uma rede cônica de 1,5 m de comprimento, 0,48 m de diâmetro de boca, $300 \mu \mathrm{m}$ de tamanho de malha e equipada com fluxômetro. A rede foi rebocada sempre no sentido contrário a corrente de deriva para evitar evasão, e com imediata fixação das amostras em solução de formalina a $4 \quad \%$ neutralizada. Paralelamente, foram obtidos dados de temperatura por termômetro de coluna de mercúrio $\left(1^{\circ} \mathrm{C}\right.$ de precisão) e salinidade da água medida por refratômetro American Optical (1 de salinidade de precisão).

A identificação dos estágios de maturação de $S$. friderici seguiram os critérios de Alvariño (1969) com 4 estágios de desenvolvimento dos ovários e vesículas seminais, sendo acrescentado o estágio zero (0) em que os indivíduos não apresentaram vestígios destas estruturas reprodutivas. Para as contagens e identificação dos estágios de desenvolvimento foi analisado todo o conteúdo das amostras.

Medidas de comprimento total dos exemplares de $S$. friderici, distância entre a extremidade da cabeça e da cauda, sem considerar a nadadeira caudal, foram tomadas com o auxílio de microscópio estereoscópico binocular com oculares micrométricas. Correlações entre a temperatura e o comprimento dos exemplares de $S$. friderici, classificados nos diferentes estágios de desenvolvimento, ocorrentes entre outubro de 1990 a abril de 1992, foram realizados após a normalização dos dados mediante a sua transformação em Log na base 10.

Para a distribuição de frequência das classes de comprimento, os organismos foram agrupados em intervalos de 0,5 $\mathrm{mm}$ de comprimento para melhor delimitação entre os estágios de desenvolvimento. Com a distribuição da freqüência, foi estimado o tempo de desenvolvimento da espécie, adotando-se como critério a ocorrência do estágio IV (em processo de reprodução) seguido pela ocorrência do estágio zero (0) ou organismos mais jovens da população. Para esta análise, só foram consideradas as amostras com ocorrência de mais de um estágio de desenvolvimento compreendidas entre novembro de
1990 à outubro de 1991, período em que as amostragens respeitaram o intervalo de 15 dias entre as coletas. Este tempo de desenvolvimento só pode ser considerado como uma aproximação, pois a sobreposição de diferentes coortes ao longo do ano dificulta este tipo de estimativa. Entretanto, uma técnica similar foi utilizada por Daponte et al. (2004) para a mesma espécie na costa da Argentina.

Para a biomassa individual foi obtida uma correlação entre o peso seco e o comprimento dos organismos em uma regressão alométrica. A obtenção do peso seco seguiu os critérios propostos por Beers (1976). Para isto, obteve-se o comprimento médio de um conjunto de 5 a 8 organismos de mesmo estágio de desenvolvimento, que foram lavados em água destilada, secos a $60{ }^{\circ} \mathrm{C}$ por 24 horas, pesados e estimado o peso seco médio individual.

Taxas de incrementos em comprimento pelo tempo foram estimados a partir das prováveis coortes identificadas ao longo do período amostral. Da mesma forma, foram estimadas as taxas de crescimento exponencial segundo a equação proposta por Omori \& Ikeda (1984):

$g=\frac{\ln W_{i}-\ln W_{i-1}}{t}$

sendo:

$\mathrm{g}=$ taxa de crescimento exponencial

$\mathrm{W}_{\mathrm{i}}$ = peso do organismo no estágio IV

$\mathrm{W}_{\mathrm{i}-1}=$ peso do organismo no estágio zero $(0)$

$\mathrm{t}$ = tempo, em dias, entre os estágios de desenvolvimento envolvidos.

\section{RESULTADOS}

Durante o período estudado observou-se uma sazonalidade tanto em temperatura como em salinidade para a água da zona de arrebentação e com repetição entre os anos de coleta (Fig. 2). Entre os meses de dezembro a abril foram observados altos valores de temperaturas (máximo de $26{ }^{\circ} \mathrm{C}$ ) e de salinidades (máximo de 36,5 ), enquanto que entre maio a novembro os baixo valores de temperatura e salinidade prevaleceram (salinidade mínima de 25 e temperatura mínima de $12^{\circ} \mathrm{C}$ ). 


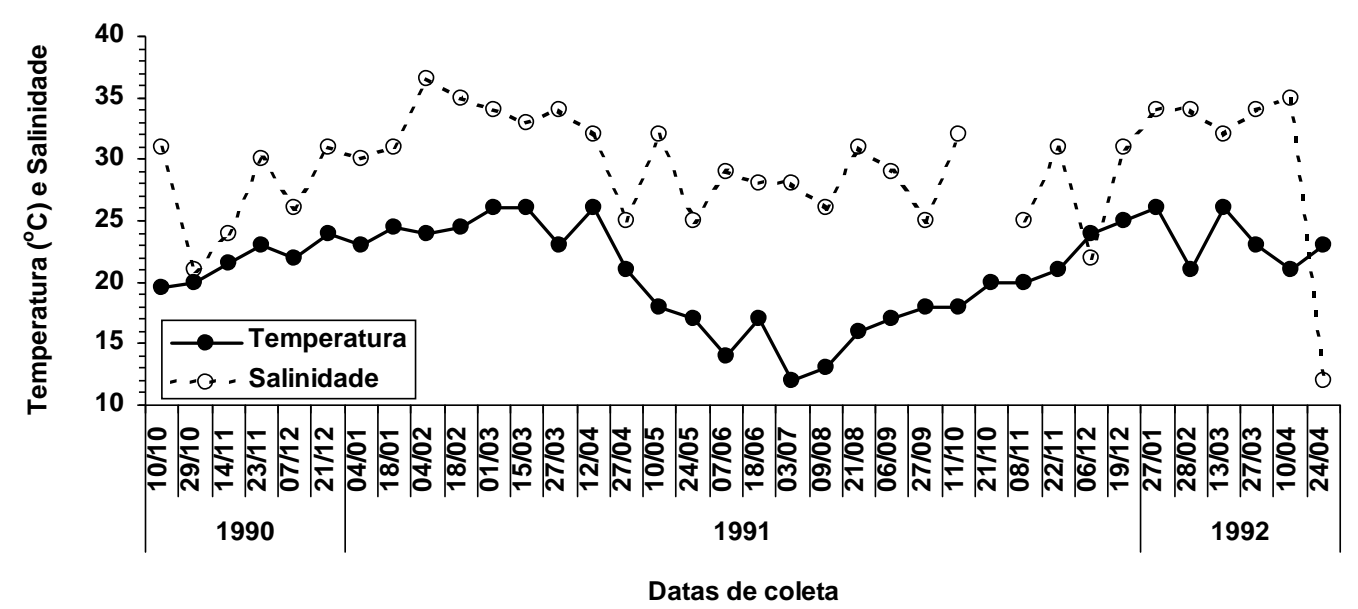

Figura 2 - Variação da salinidade $(\odot)$ e da temperatura $(\bullet)$ em ${ }^{\circ} \mathrm{C}$ durante o período amostral.

As características populacionais de S. friderici também mostraram um padrão de variação sazonal. A densidade da espécie apresentou 3 picos de ocorrência durante o ano de 1991, nos meses de fim de primavera e início de verão (1990/1991 e
1991/1992), fim do verão e no outono com um pico de densidade (1.398 Org. $\mathrm{m}^{-3}$ ) no mês de maio (Fig. 3). As altas densidades e frequência de ocorrência dos estágios de desenvolvimento I e II indicaram reprodução contínua ao longo do ano (Tabela 1).

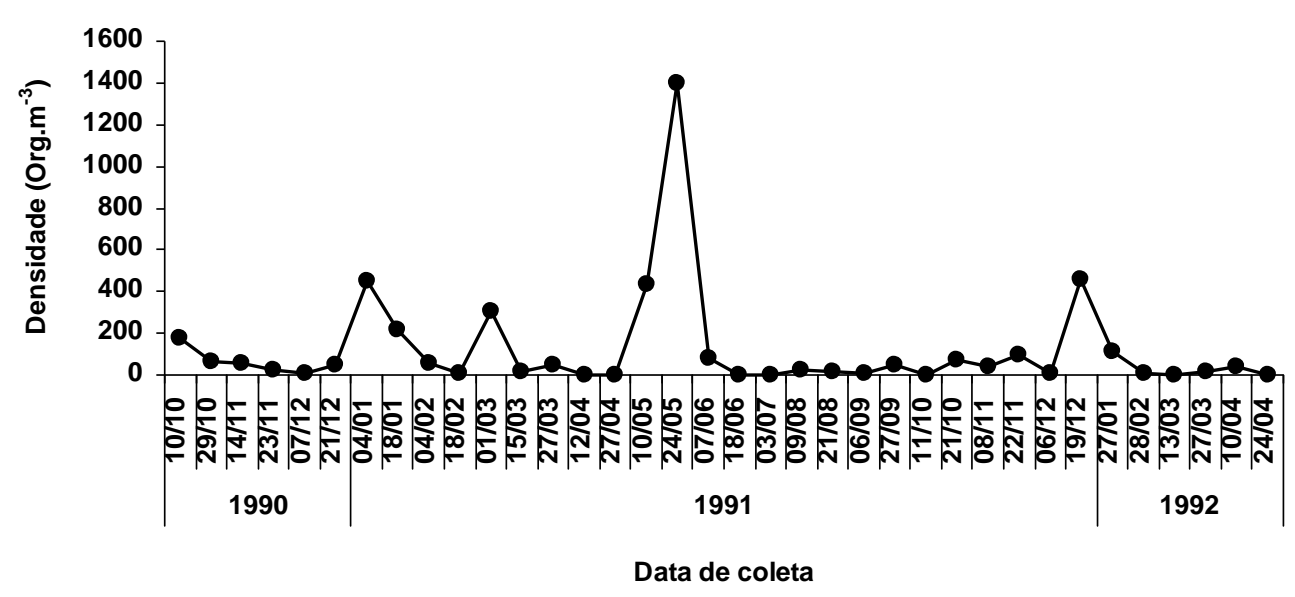

Figura 3 - Variação da densidade de S. friderici $\left(\operatorname{Org} \cdot \mathrm{m}^{-3}\right)$ durante o período amostral.

Tabela 1 - Densidade média $\left(O r g \cdot \mathrm{m}^{-3}\right.$ ) e Freqüência de Ocorrência (\%) dos estágios de desenvolvimento de Sagitta friderici em 36 amostras obtidas na zona de arrebentação na praia do Cassino (RS).

\begin{tabular}{lccccc}
\hline & \multicolumn{5}{c}{ Estágios de desenvolvimento } \\
\hline & 0 & I & II & III & IV \\
Densidade média & 14,43 & 75,60 & 33,98 & 18,74 & 15,44 \\
Freqüência de ocorrência & 38,89 & 91,67 & 75,00 & 58,33 & 61,11 \\
\hline
\end{tabular}

A variação da biomassa total da população, obtidas a partir da relação peso $X$ comprimento dos organismos (Fig. 4) e os dados de densidade de cada estágio de desenvolvimento apresentaram um comportamento similar às variações de densidade total (Fig. 3), com valores entre 0,004 a $56,76 \mathrm{mg} \cdot \mathrm{m}^{-3}$ e média de $4,31 \mathrm{mg} \cdot \mathrm{m}^{-3}$ em peso seco. 

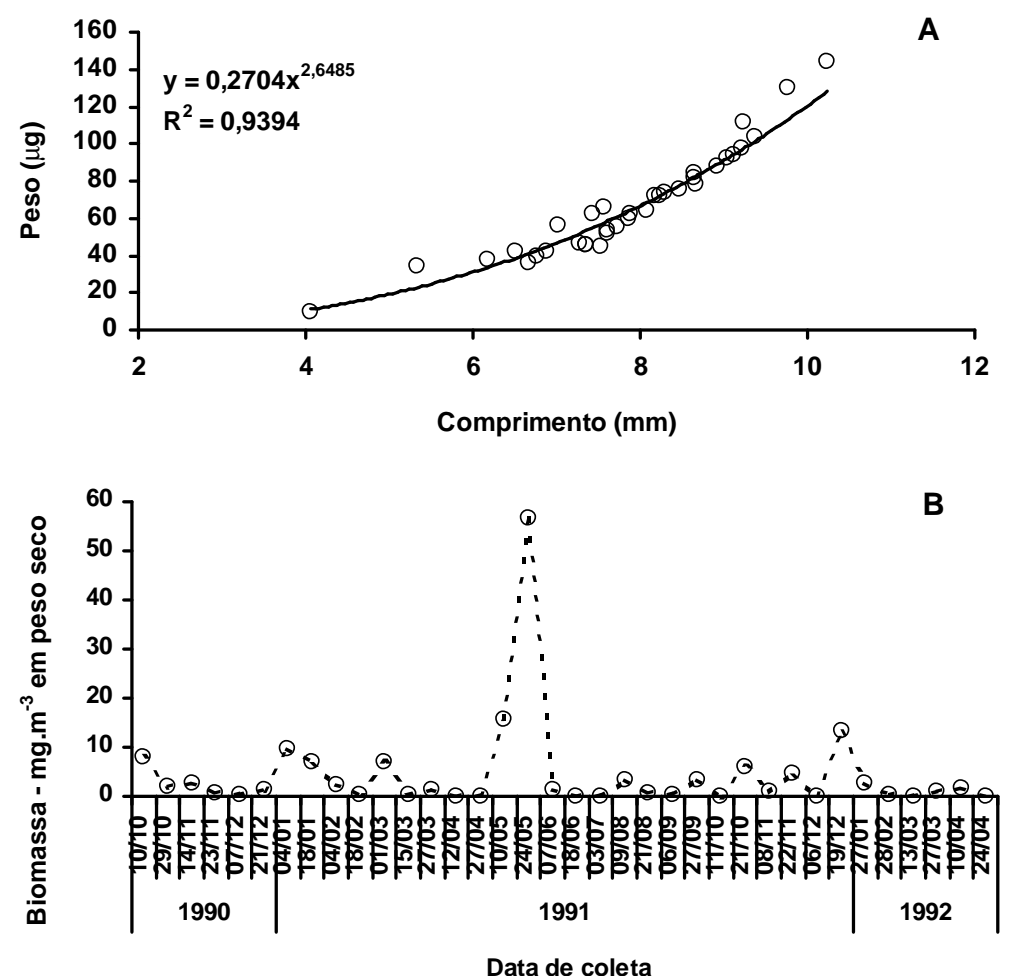

Figura 4 - A. Relação entre o comprimento $(\mathrm{mm})$ e peso seco $(\mathrm{mg})$ para $S$. friderici e B. variação da biomassa populacional $\left(\mathrm{mg}_{\mathrm{m}} \mathrm{m}^{-3}\right.$ ) de $\mathrm{S}$. friderici, em peso seco, durante o período amostral obtido pela densidade dos diferentes estágios de desenvolvimento e seu peso individual obtido pela relação comprimento peso.

Morfologicamente, o comprimento dos correlação inversa e significativa com a temperatura exemplares de $S$. friderici apresentaram uma nítida da água (Tabela 2) para os estágios de oscilação entre os meses de coleta (Fig. 5) e com desenvolvimento mais avançados.

Tabela 2 - Valores de $R^{2}$, nível de significância e número de casos (n) para as correlações entre temperatura da água e o comprimento médio para cada um dos cinco estágios de desenvolvimento de Sagitta friderici ocorrentes entre outubro de 1990 a abril de 1992 na zona de arrebentação da praia do Cassino, RS, Brasil.

\begin{tabular}{lccc}
\hline $\begin{array}{l}\text { Estágio de } \\
\text { desenvolvimento }\end{array}$ & $\mathrm{R}^{2}$ & $\begin{array}{c}\text { Nível de } \\
\text { significância }\end{array}$ & $\mathrm{n}$ \\
\hline O & 0,02 & 0,5943 & 21 \\
I & 0,30 & 0,0008 & 34 \\
II & 0,31 & 0,0006 & 34 \\
III & 0,34 & 0,0016 & 27 \\
IV & 0,45 & 0,0004 & 24
\end{tabular}



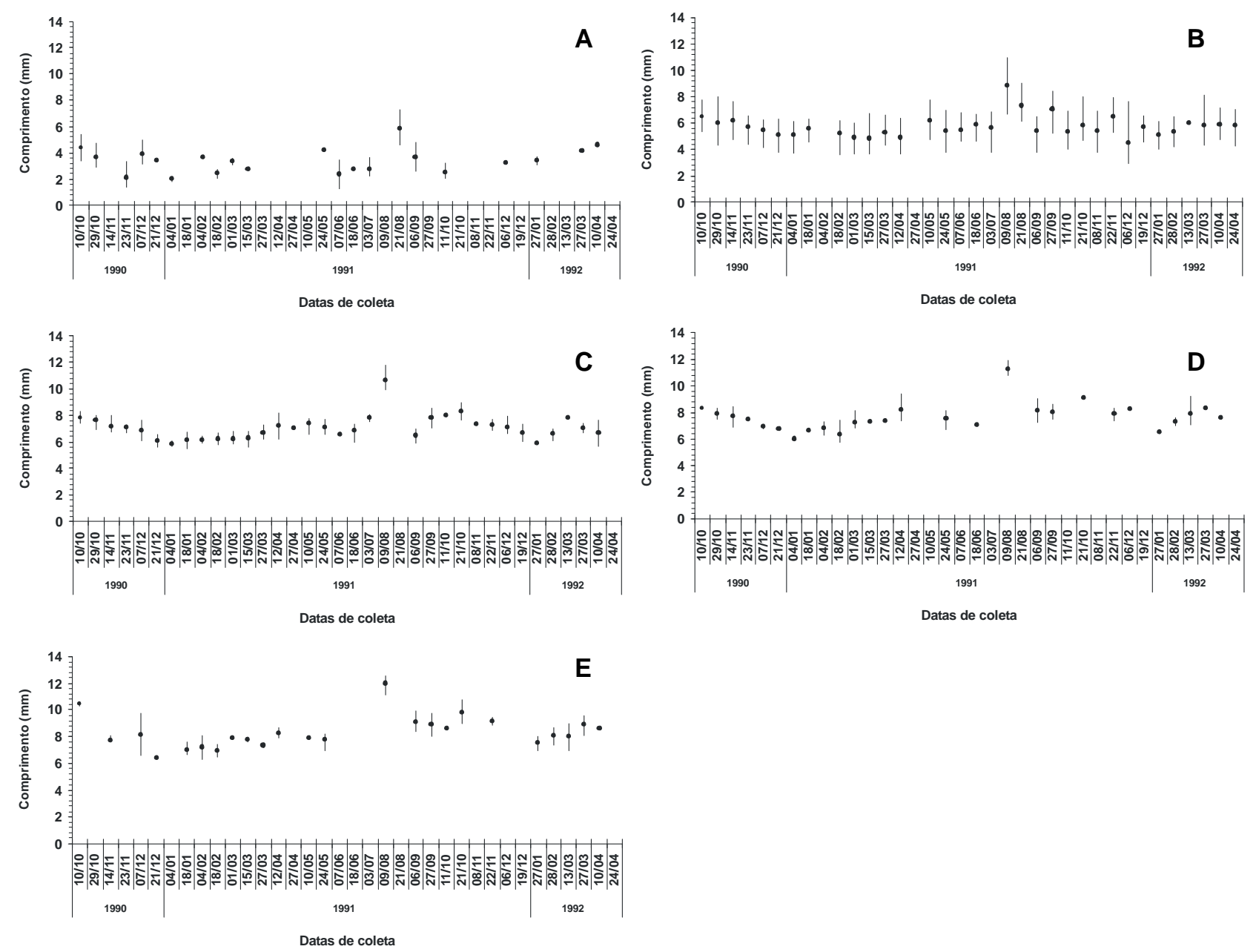

Figura 5 - Variação sazonal dos comprimentos $(\mathrm{mm})$ de $S$. friderici classificadas nos diferentes estágios de desenvolvimento. Onde: o ponto é a média e a linha vertical o intervalo dos valores máximos e mínimos. A Estágio 0; B - Estágio I; C - Estágio II; D - Estágio III; E - Estágio IV.

Em função da distribuição das classes de comprimento e dos critérios adotados de ocorrência seqüencial entre organismos no último estágio de maturação (IV) e imaturos (0) foi sugerida a ocorrência de 3 coortes por ano para $S$. friderici (Fig. 6). Estas coortes, identificadas como coortes de Primavera/Verão, Verão/Outono e Outono/Primavera apresentaram um tempo de desenvolvimento de 73 ,
95 e 136 dias respectivamente, e com uma relação inversa com a temperatura média para cada período (Tabela 3). A taxa de crescimento instantânea foi alta $(0,044)$ para a coorte de primavera/verão e baixa para as coortes de verão/outono $(0,033)$ e outono/primavera $(0,028)$. O mesmo foi observado para o incremento em comprimento.

Tabela 3 - Características populacionais de três coortes prováveis de Sagitta friderici identificadas entre 10/10/1990 a 24/04/1992 em base da ocorrência dos estágios de desenvolvimento IV seguida pelo estágio zero (0).

\begin{tabular}{lcccccc}
\hline Coorte & \multicolumn{2}{c}{ Primavera-Verão } & \multicolumn{2}{c}{ Verão-Outono } & \multicolumn{2}{c}{ Outono-Primavera } \\
\hline Data & $23 / 11 / 90$ & $04 / 02 / 91$ & $18 / 02 / 91$ & $24 / 05 / 91$ & $07 / 06 / 91$ & $21 / 10 / 91$ \\
Estágio de desenvolvimento & 0 & IV & 0 & IV & 0 & IV \\
Comprimento médio e desvio padrão $(\mathrm{mm})$ & $2,13 \pm 0,61$ & $7,20 \pm 0,49$ & $2,40 \pm 0,34$ & $7,74 \pm 0,52$ & $2,36 \pm 0,82$ & $9,80 \pm 0,60$ \\
Tempo de desenvolvimento (dias) & \multicolumn{2}{c}{73} & \multicolumn{2}{c}{95} & \multicolumn{2}{c}{136}
\end{tabular}


POPULAÇÃO DE S. FRIDERICINA PRAIA DO CASSINO

continuação

\begin{tabular}{|c|c|c|c|c|c|c|}
\hline \multirow{2}{*}{$\begin{array}{l}\text { Coorte } \\
\text { Data }\end{array}$} & \multicolumn{2}{|c|}{ Primavera-Verão } & \multicolumn{2}{|c|}{ Verão-Outono } & \multicolumn{2}{|c|}{ Outono-Primavera } \\
\hline & $23 / 11 / 90$ & $04 / 02 / 91$ & $18 / 02 / 91$ & $24 / 05 / 91$ & $07 / 06 / 91$ & $21 / 10 / 91$ \\
\hline Temperatura média e desvio padrão $\left({ }^{\circ} \mathrm{C}\right)$ & \multicolumn{2}{|c|}{$23,42 \pm 0,92$} & \multicolumn{2}{|c|}{$22,69 \pm 3,65$} & \multicolumn{2}{|c|}{$16,11 \pm 2,62$} \\
\hline $\begin{array}{l}\text { Peso }(\mathrm{mg}) \\
\text { Peso }=0,2704 \times \mathrm{Compr}^{2,6485}\end{array}$ & 2,00 & 50,42 & 2,75 & 61,07 & 2,63 & 104,10 \\
\hline Incremento em comprimento (mm/dia) & \multicolumn{2}{|c|}{0,069} & \multicolumn{2}{|c|}{0,056} & \multicolumn{2}{|c|}{0,055} \\
\hline Taxa de crescimento instantânea & \multicolumn{2}{|c|}{0,044} & \multicolumn{2}{|c|}{0,033} & \multicolumn{2}{|c|}{0,028} \\
\hline
\end{tabular}

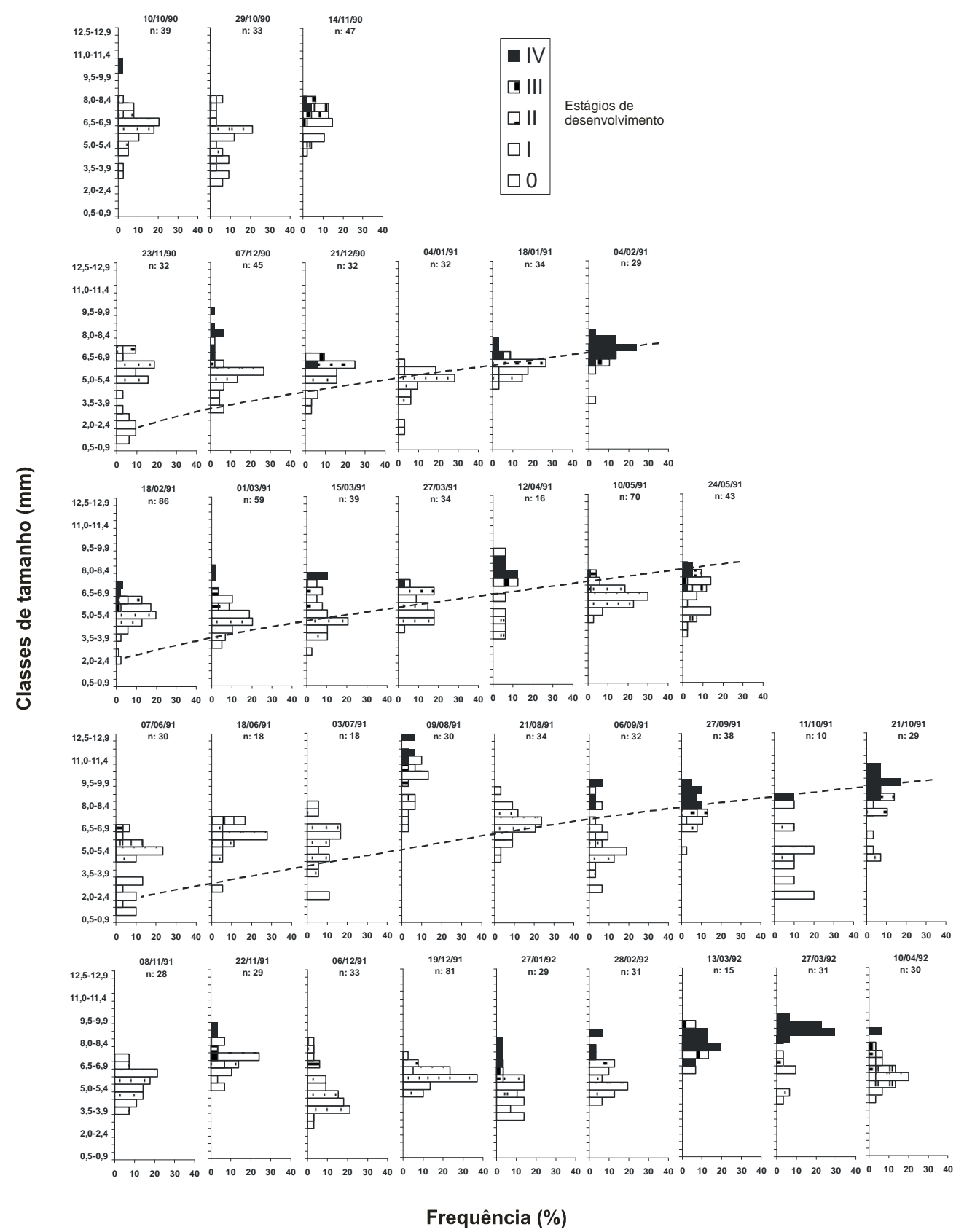

Figura 6 - Distribuição de frequência das classes de tamanho $(\mathrm{mm})$ de $S$. friderici em cada período amostral e classificados segundo os estágios de desenvolvimento de 0 a IV. As linhas tracejadas referem-se as prováveis coortes de primavera/verão, verão/outono e outono/primavera em base da ocorrência de estágio IV seguida por estágio 0. 


\section{DISCUSSÃO}

A correspondência entre as variações de densidade e do comprimento de $S$. friderici (Figs. 3 e 5) e as variações de temperatura, sugere que este parâmetro seja o controlador das características populacionais da espécie. Segundo Pearre Jr. (1991) entre outros, o comprimento e a abundância de Chaetognatha são controladas pela temperatura e disponibilidade de alimento, principalmente de copépodo. Segundo Bersano (1994), as maiores densidades de copépodes na zona de arrebentação da praia do Cassino foram registradas no verão, indicando que o alimento não é responsável pelas variações observadas.

Por outro lado, a ocorrência de grandes exemplares de $S$. friderici em agosto de 1991 sugere representantes da população da costa Argentina na área de estudo, já que os comprimentos registrados (11,11 a 12,56 mm para o estágio IV) estão dentro do intervalo de comprimento reportado por Daponte et al. (2004). Este fato sugere, mais uma vez, a influência da frente do Prata na área de estudo como destacado por Piola et al. (2000) em um estudo de caracterização das massas de água no Atlântico sul. Estes exemplares (amostra de 09/08/1991), pertecentes a populações de águas mais frias e transportados para a costa sul do Brasil, não foram considerados nas estimativas de tempo de desenvolvimento para a população residente na área de estudo.

Para a biomassa, os valores médios observados foram inferiores aos apresentados por Liang et al. (2003) para S. friderici no estuário de Cananéia, litoral de São Paulo. Para estes autores, a biomassa de Chaetognatha é maior que $50 \%$ do zooplâncton total em condições de domínio de copépodes. Entretanto, para a zona de arrebentação da praia do Cassino, o zooplâncton é dominado por Mysidacea (Bersano 1994). Em base dos dados apresentados por deste autor e as estimativas de biomassa apresentadas neste trabalho, Chaetognatha representa uma média de 3,5\% da biomassa total do zooplâncton na praia do Cassino.

A identificação e estimação de parâmetros populacionais de organismos zooplanctônicos, em base de amostras de campo, apresenta dificuldades devido a sobreposição de coortes ocorrentes em um mesmo período amostral. Esta característica é típica de ambientes de águas quentes, uma vez que em regiões polares e semi-polares são observadas poucas gerações por ano (Pearre Jr. 1991). A reprodução contínua de $S$. friderici pode ser confirmada pela ocorrência e dominância dos estágios de desenvolvimento I e II na população amostrada (Tabela 1). Análise das modas das distribuições de freqüência de comprimento dos organismos foram realizadas no presente trabalho e não resultaram na identificação confiáveis de coortes como é frequentemente citado por outros autores em estudos com Chaetognatha (Sameoto 1971, 1973, Zo 1973 e Newbury 1978). Entretanto, a análise conjunta da distruibuição dos picos de densidade, distribuição da ocorrência de adultos (Estágio IV) seguida pela ocorrência de juvenis (Estágio 0) e informações sobre a estratégia de vida dos organismos que apresentam apenas uma fase de liberação de ovos (morrendo em seguida), permitiu sugerir a existência de 3 coortes ao longo do ano na costa do Rio Grande do Sul.

Para o estuário de Cananéia, litoral de São Paulo, Liang et al. (2003) também observaram a existência de 3 coortes de $S$. friderici ao longo do ano, mas distribuídas em 4 meses de intervalo. Esta homogeneidade nas coortes pode ser devida aos altos valores de temperatura na água do ambiente $(20,3$ a $27,7^{\circ} \mathrm{C}$ ). Estes autores também observaram 3 picos de densidade da população e variação de comprimento dos diferentes estágios de desenvolvimento apesar da discreta sazonalidade da temperatura.

$\mathrm{Na}$ costa da Argentina, Daponte et al. (2004) cita a duração no desenvolvimento de $S$. friderici de 249 dias com um incremento de $0,03 \mathrm{~mm}$.dia- 1 à uma variação de temperatura de 10,1 a $21,1 \stackrel{\circ}{\circ}$. Para este caso, os adultos atingiram $15,1 \mathrm{~mm}$ de comprimento total, ou seja, comprimento e tempo de desenvolvimento bem superiores aos registrados no presente trabalho. Neste mesmo trabalho, os autores observaram que o comprimento final dos adultos de $S$. friderici também estiveram inversamente correlacionados com a temperatura da água. $\mathrm{Na}$ costa do Marrocos, Furnestin (1957) observou o comprimento máximo de $13 \mathrm{~mm}$ para $S$. friderici em água cuja temperatura média foi de $18,0^{\circ} \mathrm{C}$.

Como uma espécie eurihalina e euritérmica (Resgalla Jr. \& Montú 1995), S. friderici apresenta maior 
adaptação em águas costeiras que outras espécies de Chaetognatha. Sua taxa de alimentação atribui uma posição de forte competidor no nível dos consumidores secundários como destacado por Vega-Pérez \& Liang (1992) e Liang et al. (2003) em base dos hábitos alimentares da espécie. Associado a isto, e de acordo com os resultados obtidos, apresenta um ciclo de vida relativamente curto e com potencial de 3 coortes por

\section{LITERATURA CITADA}

ALVARIÑO, A. 1969. Los quetognatos del Atlantico. Distribucion y notas esenciales de sistemática. Inst. Español Oceanogr. Madrid. 289pp.

BEERS, JR. 1976. Determination of zooplankton biomass. In: STEEDMAN, HF (ed.). Zooplankton fixation and preservation. Monographs on Oceanographic Methodology. UNESCO, Paris. 35-86.

BERSANO, JG. 1994. Zooplâncton da zona de arrebentação, de praias arenosas situadas ao sul do município de Rio Grande RS, Primavera 1990 - Verão 1991. Tese de Mestrado, Univ. Rio Grande. 163p.

BOLTOVSKOY, D. 1981. Chaetognatha. In: BOLTOVSKOY, D (ed.). Atlas del zooplancton del Atlántico Sudoccidental y métodos de trabajo con el zooplancton marino. Publ. Espcial INIDEP, Mar del Plata, Argentina. 759-792p.

BONE, Q, H KAPP \& AC PIERROT-BULTS. 1991. Introduction and relationships of the group. In: BONE, $Q, H$ KAPP \& AC PIERROT-BULTS (eds.). The Biology of Chaetognaths. Oxford Univesity Press, Oxford. 1-4.

BORZONE, CA. 1988. Estudo da macrofuna bentônica infralitoral da região costeira adjascente à barra do Rio Grande, RS, Brasil. Dissertação de Mestrado. FURG. 113p.

CALLIARI, L. 1997. Coastal and marine environments and their biota: geological setting. In: SEELINGER, U, C ODEBRECHT \& JP CASTELLO (eds.) Subtropical convergence environments: The coast and sea in the southwestern atlantic. SpringerVerlag, Heidelberg. 91-94.

CASTELLO, JP \& OO MÖLLER Jr. 1977. Sobre as condições oceanográficas do Rio Grande do Sul. Atlântica, Rio Grande. 2(2):25-110.

CAVALIERI, F. 1963. Nota preliminar sobre Sagitta (Chaetognatha) del litoral Atlántico Argentino. Physis, 24(67):223-236.

DAPONTE, MC, FL CAPITANIO, DE NAHABEDIAN, MD VIÑAS \& RM NEGRI. 2004. Sagitta friderici Ritter-Záhony (Chaetognatha) from south Atlantic waters: abundance, population structure, and life cycle. J. Mar. Sci. 61:680-686.

FURNESTIN, M-L. 1957. Chaetognathes et zooplankton du secteur Atlantique Marocain. Revue des Travaux de I'Institut des Pêches Maritimes. 21:1-356.

LIANG, T-H, K ARA, LB MIRANDA, AL BÉRGAMO \& $M$ BERNADES. 2003. On the variability of the chaetognath Sagitta friderici Ritter-Zàhony at Cananéia Lagoon Estuary system, São Paulo, Brazil. Hydrobiologia, 510:91-102. ano nas regiões sudeste e sul. Estas características conferem, à espécie, dominância e consequentemente importância na ecologia pelágica da costa brasileira.

\section{AGRADECIMENTOS}

Ao Dr. José Guilherme Bersano Filho pela disponibilidade das amostras de zooplâncton da praia do Cassino. Bolsa PQ-CNPq processo 302165/2008-8.

LOPES, RM. 2007. Marine zooplankton studies in Brazil - A brief evaluation and perspectives. An. Acad. Bras. Cienc., 79(3):369-379.

MONTÚ, M. 1980. Zooplâncton do Estuário da Lagoa dos Patos. I. Estrutura e variações temporais espaciais da comunidade. Atlântica, Rio Grande. 4: 53-72.

MONTÚ, M \& TA CORDEIRO. 1988. Zooplâncton del complejo estuarial de la Bahía de Paranaguá. I. Composición, dinámica de las especies, ritmos reproductivos y acción de los factores aqmbientales sobre la comunidad. Nerítica 3(1):61-83.

NEWBURY, TK. 1978. Comsuption and growth rates of chaetognaths and copepods in subtropical oceanic waters. Pac. Sc. 32(1):61-78.

OMORI, M \& T IKEDA. 1984. Methods in marine zooplankton ecology. John Wiley \& Sons Publ. 332p.

PEARRE Jr., S. 1991. Growth and reproduction. In: BONE, Q, H KAPP \& AC PIERROT-BULTS (eds.). The biology of Chaetognaths. Oxford University Press, Oxford, 61-75.

PIOLA, AR. EJD CAMPOS, OO MÖLLER Jr., M CHARO \& C MARTINEZ. 2000. Sub-tropical shelf front off eastern south America. J. Geophys. Res. 105(C3): 6565-6578.

RAMIREZ, FC \& MD VIÑAS. 1982. Variacion estacional de los estados gonadales y las frecuencias de talla en Sagitta friderici, un quetognato neritico del area de Mar del Plata. Physis, Secc. A, 41(100):99-111.

RESGALLA Jr., C. 2008. Pteropoda, Cladocera and Chaetognatha associations as hydrological indicators in the Southern Brazilian Shelf. Latim Am. J. Aquat. Res. 36(2):271-282.

RESGALLA Jr., C \& M MONTÚ. 1995. Quetognatos de la plataforma continental del sur de Brasil. Inv. Mar. CICIMAR, México. 10:23-41.

RESGALLA Jr., C, VG COUTINHO DE SOUZA, LR RORIG \& CAF SCHETTINI. 2008. Spatial and temporal variation of the zooplancton community in the area of influence of the Itajaí-açu river, SC (Brazil). Braz. J. Oceanog. 56(3):211-224.

SAMEOTO, DD. 1971. Life history, ecological production, and an empirical mathematical model of the population of Sagitta elegans in St. Margaret's Bay, Nova Scotia. J. Fish. Res. Boa. Canada, 28(7):971-985.

SAMEOTO, DD. 1973. Annual life cycle and production of the chaetognath Sagitta elegans in Bedford Basin, Nova Scotia. J. Fish. Res. Boa. Canada. 30(3):333-344.

VEGA-PÉREZ, AA \& TH LIANG. 1992. Feeding of a pelagic chaetognatha, Sagitta friderici Ritter-Záhony, off Ubatuba region (São Paulo, Brazil). Bol. Inst. Oceanogr. 40:93-100.

ZO, Z. 1973. Breeding and growth of the chaetognath Sagitta elegans in Bedford Basin. Limn. Oceanogr. 18(5):750-756.

Recebido - 05/02/2009

Aceito: 02/05/2010 
